\title{
Role of MiR-98 and Its Underlying Mechanisms in Systemic Lupus Erythematosus
}

\author{
Lin Xie $\square$ and Jinhua Xu
}

ABSTRACT. Objective. T-lymphocyte apoptosis plays a critical role in the pathogenesis of systemic lupus erythematosus (SLE). However, the underlying regulatory mechanisms of apoptosis in SLE remain unclear. The aim of this study was to explore the role of miR-98 in SLE and its underlying mechanisms.

Methods. Western blotting and quantitative reverse transcription PCR (qRT-PCR) were used to analyze miR-98 and Fas expression. Luciferase reporter assays were performed to identify miR-98 targets. To modify miRNA levels, miR-98 mimics and inhibitor were transfected into cells. A lentiviral construct was used to overexpress the level of Fas in SLE CD4+ T cells. Gene and protein expression were determined by qRT-PCR and Western blotting. Apoptosis levels were evaluated by annexin V staining and flow cytometry.

Results. Compared to those of healthy donors, miR-98 was downregulated in SLE CD4+ T cells, whereas Fas mRNA and protein expression were upregulated. Upregulation of miR-98 by mimic transfection protected Jurkat cells against Fas-mediated apoptosis at both mRNA and protein levels, while miR-98 inhibitor induced the completely opposite effect. Luciferase reporter assays demonstrated that miR-98 directly targeted Fas mRNA. Further, miR-98 inhibitor induced apoptosis in primary healthy CD4+ T cells through the Fas-caspase axis, while upregulation of miR-98 in SLE CD4+ T cells led to the opposite effect.

Conclusion. The current study revealed that downregulation of miR-98 induces apoptosis by modulating the Fas-mediated apoptotic signaling pathway in SLE CD4+ T cells. These results suggest that miR-98 might serve as a potential target for SLE treatment. (First Release June 15 2018; J Rheumatol 2018;45:1397-1405; doi:10.3899/jrheum.171290)

Key Indexing Terms:

miR-98 FAS APOPTOSIS CD4+ T CELLS SYSTEMIC LUPUS ERYTHEMATOSUS

Systemic lupus erythematosus (SLE) is a multisystem autoimmune disease characterized by abnormal T-lymphocyte activation and high-titer autoantibody production that triggers inflammation and causes widespread tissue and organ damage ${ }^{1}$. These pathogenic autoantibodies are produced by plasma cells differentiated from activated autoreactive B cells. As a T-lymphocyte subset, CD4+ T cells provide "help" to B cells to produce autoantibodies and promote inflammation, and is reported to be the predominant helper subset and the strongest inducer of autoantibodies ${ }^{2}$. In addition, CD4+ T cells are considered pivotal in the development and maintenance of protective immunity and tolerance, and imbalances in these processes can lead to autoimmunity ${ }^{3,4}$. Although many defects in signaling and functions have been identified in the immune cells of SLE,

From the Department of Dermatology, Huashan Hospital, Fudan University, Shanghai, China.

Supported by grants from the National Natural Science Foundation of China (No.81373212) and from Shanghai Municipal Commission of Health and Family Planning (No. 2017ZZ02002).

L. Xie, MD, PhD; J. Xu, MD, PhD, Professor, Department of Dermatology, Huashan Hospital, Fudan University.

Address correspondence to Dr. J. Xu, Department of Dermatology,

Huashan Hospital, Fudan University, No. 12 Middle Wulumuqi Road,

Shanghai 200040,China.E-mail:xjhhsyy@163.com

Accepted for publication April 30, 2018. the majority of them is in CD4+ T cells; however, the mechanism is not well clarified ${ }^{5}$.

The formation of autoantibodies is thought to be the result of alterations of cell death pathways, including apoptosis, necrosis, neutrophil extracellular trap formation (called NETosis), and increase of low-density granulocyte generation $^{6,7}$. Among these, T-lymphocyte apoptosis plays an important role in the pathogenesis of $\mathrm{SLE}^{8}$. Either enhanced apoptosis or defective clearance of apoptotic cells may lead to increased exposure of nucleosomes to the immune system $^{9}$. Patients with SLE exhibit increased levels of apoptotic total T-lymphocytes and CD4+ $\mathrm{T}$ cells ${ }^{10,11,12}$. Moreover, increased apoptosis correlates with SLE disease activity and might be responsible for reduced $\mathrm{T}$ cell frequency ${ }^{11,12}$. The increased production of apoptotic cells may result from increased lymphocyte activation ${ }^{13}$ or be triggered by autoantibodies such as antiphosphatidylserine antibodies $^{14}$ or anti-RNP ${ }^{15}$. However, the mechanisms governing the regulation of apoptosis in SLE remain elusive.

The major route for apoptosis induction in activated lymphocytes is through Fas ${ }^{16}$. Fas, also known as APO-1 or CD95, is a membrane receptor expressed by multiple cell types ${ }^{17}$, whereas its ligand, Fas ligand (FasL), a membrane glycoprotein, is expressed on activated $\mathrm{T}$ cells. The cross-linking of Fas by FasL triggers apoptotic cell death with

Personal non-commercial use only. The Journal of Rheumatology Copyright $\odot$ 2018. All rights reserved. 
characteristic cytoplasmic and nuclear condensation, as well as DNA fragmentation ${ }^{18}$. The FasL/Fas system has been well described in the establishment and maintenance of peripheral tolerance ${ }^{19}$. Indeed, defects in either Fas or FasL in mice result in acceleration of SLE-like diseases ${ }^{17}$. Increased FasL/Fas expression and caspase- 3 activity together with subsequent increases in T-lymphocyte cell apoptosis (particularly in CD4+ T cells) are detected in human SLE $20,21,22$. There is also evidence for modulation of the FasL/Fas system by secretion of soluble Fas in patients with SLE $^{23,24}$. Moreover, patients with juvenile-onset SLE exhibit upregulation of apoptosis-related protein Bcl-2 and Fas in total $\mathrm{T}$-lymphocytes and CD4+ T cells ${ }^{25}$. Based on these findings, CD4+ $\mathrm{T}$ cell apoptosis may be defective in SLE, and the FasL/Fas-mediated signaling pathway likely has a substantial influence on the process. Therefore, targeted inhibition of CD4+ T cell apoptosis is a key approach to treating SLE. However, the molecular components regulating Fas-mediated apoptosis in SLE CD4+ T cells remain poorly understood.

MicroRNA (miRNA) are small noncoding RNA that regulate gene expression by acting on their target mRNA, resulting in translation inhibition or mRNA degradation. One of the first identified miRNA families, let-7/miR-98, has a great influence on cell proliferation, differentiation, apoptosis, and oncogenesis ${ }^{26}$. In HeLa cells, miR-98 targets Fas and regulates Fas-mediated apoptosis in a dose-dependent manner $^{27}$. In addition, let-7/miR-98 expression is reduced in activation-induced cell death of CD4+ T cells, accompanied by increased Fas expression ${ }^{27}$. By downregulating Fas/caspase-3 apoptotic signaling pathway, miR-98 regulates myocardial infarction-induced apoptosis ${ }^{28}$. However, the functional role of miR-98 in CD4+ T cell apoptosis in patients with SLE has not been previously investigated.

In our study, we identified the expression patterns of miR-98 in CD4+ T cells from patients with SLE and explored the potential role and underlying mechanisms of miR-98 in regulating cell apoptosis and dysfunction in SLE. Our findings suggested that Fas might be one of several distinct targets of miR-98 that contribute to its ability to regulate cell survival and death by the regulation of apoptosis.

\section{MATERIALS AND METHODS}

Subjects. A total of 48 patients with SLE, who were diagnosed based on the 1997 American College of Rheumatology classification criteria, were recruited from Huashan Hospital, Fudan University. Disease activity was assessed on the basis of the SLE Disease Activity Index (SLEDAI). Relevant clinical and laboratory information regarding the patients is summarized in Table 1. Thirty-nine age- and sex-matched healthy donors were recruited voluntarily. This study was approved by the Independent Ethics Committee of Huashan Hospital (No. 2017-101). Written informed consent was obtained from all subjects.

Cell isolation and culture. Peripheral blood mononuclear cells (PBMC) were isolated by density-gradient centrifugation, and CD4+ T cells were obtained by magnetic separation (Miltenyi Biotec). Purified CD4+ T cells were cultured in OpTmizer CTS T-Cell Expansion SFM (Gibco) supplemented
Table 1. Clinical and laboratory characteristics of patients with SLE in the study.

\begin{tabular}{|c|c|c|}
\hline Characteristics & $\begin{array}{l}\text { SLE in Figure 1, } \\
\qquad \mathrm{n}=35\end{array}$ & $\begin{array}{c}\text { SLE in Figure 5, } \\
\mathrm{n}=13\end{array}$ \\
\hline Sex, male/female, $n$ & $4 / 31$ & $1 / 12$ \\
\hline Age, yrs, median (range) & $33(15-58)$ & $30(23-58)$ \\
\hline SLEDAI score, median (range) & $6(0-16)$ & $7(0-13)$ \\
\hline $\begin{array}{l}\text { Anti-dsDNA, IU/ml, median } \\
\text { (range) }\end{array}$ & $201.7(1-800)$ & $116.9(33.5-800)$ \\
\hline C3, g/l, median (range) & $0.63(0.17-1.24)$ & $0.78(0.25-1.27)$ \\
\hline $\mathrm{C} 4, \mathrm{~g} / \mathrm{l}$, median (range) & $0.10(0.06-0.32)$ & $0.13(0.07-0.25)$ \\
\hline Steroids, $\mathrm{n}^{\mathrm{a}}$ & $30 / 5$ & $11 / 2$ \\
\hline Secondary agents, $\mathrm{n}^{\mathrm{a}, \mathrm{b}}$ & $25 / 10$ & $9 / 4$ \\
\hline
\end{tabular}

a The medications that patients received when taking blood samples. ${ }^{\mathrm{b}}$ Some patients received secondary antirheumatic agents, including methotrexate, azathioprine, cyclophosphamide, and chloroquine. SLE: systemic lupus erythematosus; SLEDAI: SLE Disease Activity Index.

with $1 \%$ L-glutamine and $1 \%$ penicillin/streptomycin. Jurkat cells (Chinese Academy of Sciences) were cultured in RPMI 1640 medium supplemented with $10 \%$ fetal bovine serum and $1 \%$ penicillin/streptomycin.

RNA extraction and quantitative reverse transcription PCR ( $q R T-P C R)$. Total RNA was extracted with TRIzol reagent (Invitrogen). Reverse transcription was performed to obtain cDNA for mRNA using PrimeScript RT Master Mix (Takara) and for miRNA using a First-strand cDNA synthesis kit (GeneCopoeia) as previously described ${ }^{29}$. SYBR Premix Ex Taq II (Takara) and an All-in-one miRNA qRT-PCR Detection kit (GeneCopoeia) were used to carry out qPCR. GAPDH or U48 were used as internal controls. The following primers were used: Fas (forward 5'-TCT GGT TCT TAC GTC TGT TGC-3', reverse $5^{\prime}$-CTG TGC AGT CCC TAG CTT TCC-3') and GAPDH (forward 5'-GGA GCG AGA TCC CTC CAA AAT-3', reverse 5'-GGC TGT TGT CAT ACT TCT CAT GG-3'). The primers for miR-98 and $\mathrm{U} 48$ were directly purchased from GeneCopoeia. All reactions were run in triplicate.

MiRNA mimic, inhibitor, and cell transfections. Cells were transiently transfected with miR-98 mimics, miR-98 inhibitor, or mimic/inhibitor controls (GenePharma) using Lipofectamine RNAiMAX (Invitrogen), and were collected for further analysis $48 \mathrm{~h}$ later. The transfection efficiency, evaluated by flow-cytometric analysis relative to a carboxyfluorescein (FAM) dyelabeled miRNA mimic/inhibitor negative control, reached $70 \%$ to $80 \%$.

Luciferase reporter assay. The 3'-untranslated region (3'-UTR) of Fas mRNA and its site-directed mutants were PCR-amplified and cloned into psiCHECK-2 vectors (Promega). Afterward, wild-type (WT) or mutated (MUT) luciferase plasmids and miR-98 mimic/negative controls were co-transfected into $293 \mathrm{~T}$ cells. Luciferase activities were assessed using a Dual-Luciferase Reporter Assay System (Promega) in accordance with the manufacturer's instructions.

Western blotting. A protein concentration assay kit was obtained from ThermoFisher. Proteins were separated on $12 \%$ sodium dodecyl sulfate-polyacrylamide gels and transferred to PVDF membranes (Millipore). The membranes were incubated with anti-Fas, anti-Cleaved Caspase-3, anti-Caspase-3, anti-Cleaved Caspase-8, anti-Caspase-8, anti-Cleaved Caspase-9, anti-Caspase-9, anti-Bax, anti-Bcl-2, and anti-GAPDH (Cell Signaling Technology) antibodies overnight at $4^{\circ} \mathrm{C}$, followed by an incubation with corresponding horseradish peroxidaseconjugated secondary antibodies, according to previously published protocols ${ }^{30}$. Detection was carried out using an enhanced chemiluminescence kit (Millipore). GAPDH was used as a loading control.

Lentiviral infection. Purified lentiviral particles containing Fas (LV-Fas) or vector control (LV-vector) and a green fluorescent protein (GFP) marker

Personal non-commercial use only. The Journal of Rheumatology Copyright $\odot$ (2018. All rights reserved 
were purchased from GENECHEM. SLE CD4+ T cells were incubated with viral particles mixed with $8 \mu \mathrm{l} / \mathrm{ml}$ polybrene following centrifugation at 1000 $\mathrm{g}$ for $1 \mathrm{~h}$. Twelve hours after infection, the cells were added to fresh complete medium and were collected for further analysis $72 \mathrm{~h}$ later. GFP was detected by fluorescence microscope after $72 \mathrm{~h}$, and the ratio of GFP protein suggested whether cells were successfully infected. The overexpression efficiency of Fas was confirmed by qRT-PCR.

Apoptosis assay. Cell apoptosis was detected by flow cytometry analysis with an Annexin V Apoptosis Detection Kit FITC (eBioscience). Briefly, cells were washed once in PBS and resuspended in binding buffer at a concentration of $2 \times 10^{6}$ cells $/ \mathrm{ml}$. Cells $(100 \mu \mathrm{l})$ were then incubated with FITC-annexin V ( $5 \mu \mathrm{l})$ for $15 \mathrm{~min}$ at room temperature and washed once in binding buffer, followed by resuspended in $200 \mu \mathrm{l}$ of binding buffer with propidium iodide $(5 \mu \mathrm{l})$. For SLE CD4+ T cells transfected with lentivirus expressing GFP spectrally overlapped between FITC-annexin V and GFP, allophycocyanin-annexin $\mathrm{V}$ in conjunction with 7-AAD was used to avoid the overlap. Data were acquired with a BD Accuri C6 or FACSCanto flow cytometer and analyzed using FlowJo software. Annexin V-positive apoptotic cells were quantified.

Statistical analysis. All data are expressed as the mean \pm SEM. GraphPad Prism software was used for statistical analysis. Variables were comparatively analyzed using Student $t$ test. Correlations were determined by Pearson correlation coefficient. For all statistical tests, a p value $<0.05$ was considered statistically significant.

\section{RESULTS}

MiR-98 is downregulated in freshly isolated CD4+ T cells from patients with SLE. Because miR-98 has been found in several studies to influence apoptotic cell death, we performed qRT-PCR analysis to determine the expression of endogenous miR-98 in samples obtained from CD4+ T cells from 20 patients with SLE and 20 healthy donors. As shown in Figure 1A, miR-98 expression was much lower in SLE CD4+ T cells than in healthy control CD4+ T cells $(p<0.01)$, suggesting a specific association between decreased miRNA expression and the aberrant CD4+ T cell function in SLE. Next, we evaluated the correlations between miR-98 expression and clinical features. A significant negative correlation was observed between miR-98 levels and SLEDAI scores $(r=-0.4305, p=0.0373$; Figure 1B). However, sex or age was not obviously associated with miR-98 expression (data not shown).

Increased expression of Fas and apoptosis in freshly isolated $C D 4+T$ cells from patients with SLE. To determine whether the expression levels of Fas in SLE CD4+ T cells are comparable to those of miR-98, we carried out qRT-PCR and Western blot analysis using total RNA and proteins isolated from CD4+ T cells from patients with SLE and healthy donors. Fas mRNA (Figure 1C) and protein (Figure 1E) expression levels were higher in SLE CD4+ T cells than in
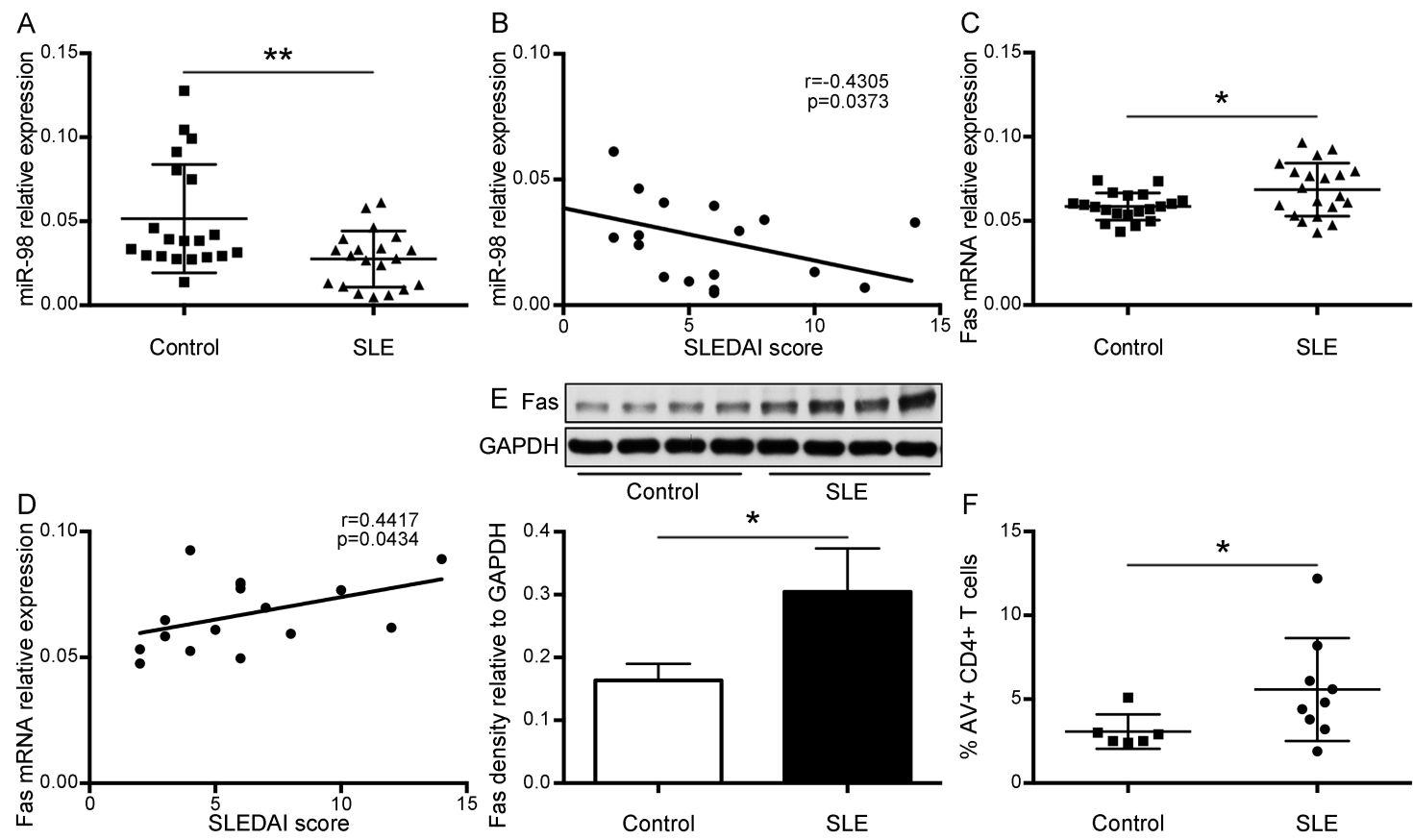

Figure 1. Expression of miR-98 and Fas in CD4+ T cells from patients with SLE and healthy donors. A. Analysis by qRT-PCR of miR-98 expression in CD4+ T cells from patients with SLE $(n=20)$ and healthy donors $(n=20)$. B. Correlation between miR-98 expression and SLEDAI scores. Relative miRNA expression was normalized to U48. C. Analysis by qRT-PCR of Fas mRNA expression in CD4+ T cells from patients with SLE $(n=20)$ and healthy donors $(n=20)$. D. Correlation between Fas mRNA expression and SLEDAI scores. Relative mRNA expression was normalized to GAPDH. E. Representative Western blot analysis of Fas protein expression in CD4+ T cells from patients with SLE $(n=9)$ and healthy donors $(n=9)$ is reported along with its relative quantification in the histogram. GAPDH was used as the loading control. F. Apoptosis levels were analyzed by measuring annexin V-positive cells using flow cytometry. Each point represents an individual patient $(n=9)$ or healthy donor $(n=6)$. Data are shown as the mean \pm SEM. $* \mathrm{p}<0.05$. ** $\mathrm{p}<0.01$. miR-98: microRNA98; SLE: systemic lupus erythematosus; qRT-PCR: quantitative reverse transcription PCR; SLEDAI: SLE Disease Activity Index.

Personal non-commercial use only. The Journal of Rheumatology Copyright (c) 2018. All rights reserved. 
healthy donors CD4+ T cells, which suggested the dependence of Fas expression on endogenous miR-98 expression in CD4+ T cells. A significant positive correlation was also observed between Fas mRNA levels and SLEDAI scores $(r=0.4417, p=0.0434$; Figure 1D). Because the major route for apoptosis induction in activated lymphocytes is through $\mathrm{Fas}^{16}$, we next examined the apoptosis levels in SLE. In line with previously published data ${ }^{11,31}$, the annexin V/PI double-staining assay showed that the percentage of freshly isolated CD4+ T cells that were apoptotic was significantly higher in patients with SLE than in healthy donors (Figure 1F). MiR-98 directly targets the 3'-UTR of Fas and downregulates Fas-mediated caspases. To validate the effect of miR-98 on Fas regulation, we examined the effect of miR-98 by transfecting Jurkat cells with miR-98 mimics or miR-98 inhibitor (anti-miR-98). Both qRT-PCR and Western blot analysis showed that transfection with miR-98 mimics significantly reduced Fas expression at mRNA and protein levels, whereas transfection with miR-98 inhibitor, which blocked endogenous miR-98, boosted Fas expression (Figure 2A-2F), indicating that the expression of Fas is negatively regulated by miR-98.

To further elucidate the functional consequences of miR-98-mediated regulation of Fas, we investigated its role in regulating the expression of proteins involved in the Fas-mediated apoptotic pathway (cleaved caspase-3 and cleaved caspase-8) and the expression of intrinsic apoptosis molecules (cleaved caspase-9, Bax, and Bcl-2). As expected, the ratio of cleaved/total caspase-3 and cleaved/total caspase- 8 expression was markedly downregulated by transfection of Jurkat cells with miR-98 mimics, whereas transfection with miR-98 inhibitor achieved the opposite effect. However, the ratio of cleaved/total caspase- 9 and the expression of Bax and Bcl-2 remained almost unchanged (Figure 2B, 2C, 2E, and 2F).

To demonstrate a direct interaction between Fas and

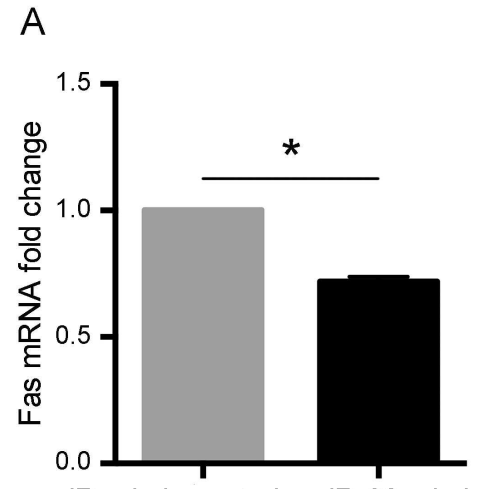

miR-mimic control miR-98 mimics

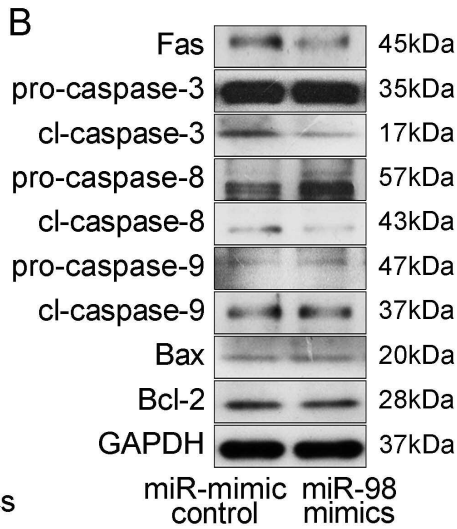

E

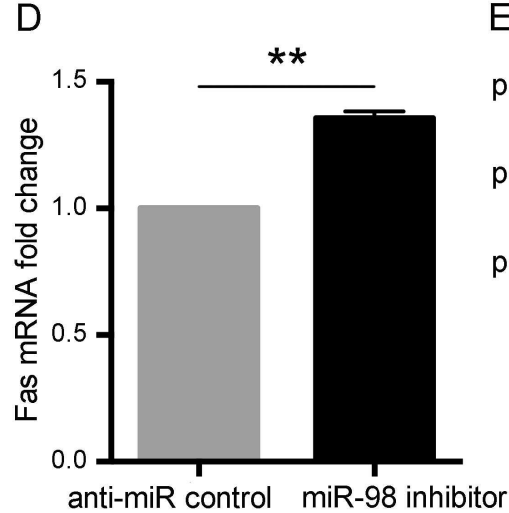

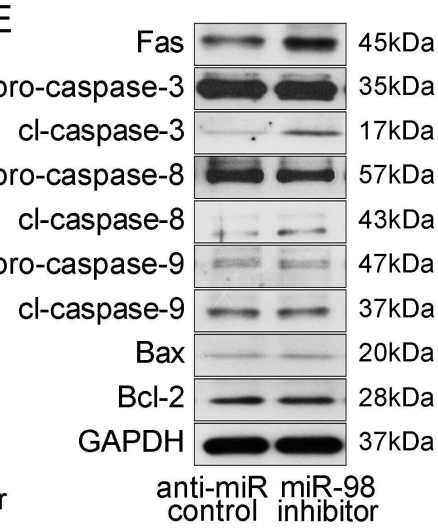
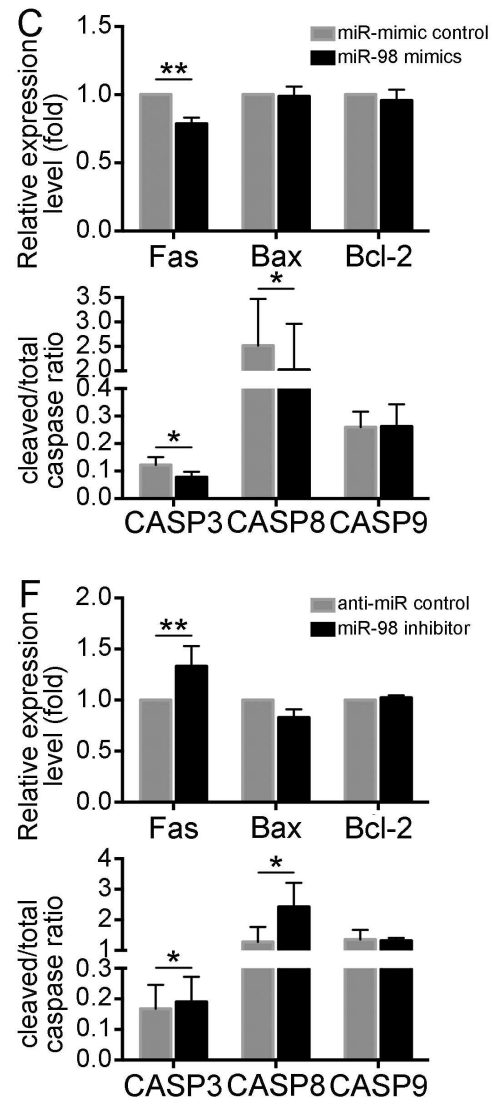

Figure 2. MiR-98 downregulates Fas-mediated caspases in Jurkat cells. A. Analysis by qRT-PCR of Fas mRNA expression in Jurkat cells $48 \mathrm{~h}$ after transfection with miR-98 mimics. Histograms show the fold changes in mRNA expression regarding controls after normalization to GAPDH. B. Western blot analysis of Fas and apoptosis-related protein expression in Jurkat cells $48 \mathrm{~h}$ after transfection with miR-98 mimics. GAPDH was used as the loading control. C and F. Histograms show the fold changes in Fas, Bax, and Bcl-2 protein expression and the ratio of cleaved/total caspase-3, 8, and 9 protein expression after transfection with miR-98 mimics or inhibitor. D. Analysis by qRT-PCR of Fas mRNA expression in Jurkat cells $48 \mathrm{~h}$ after transfection with miR-98 inhibitor. E. Western blot analysis of Fas and apoptosis-related protein expression in Jurkat cells $48 \mathrm{~h}$ after transfection with miR-98 inhibitor. Data shown are representative of 3 or more independent experiments (mean \pm SEM). $* \mathrm{p}<0.05 . * * p<0.01$. miR-98: microRNA98; qRT-PCR: quantitative reverse transcription PCR.

Personal non-commercial use only. The Journal of Rheumatology Copyright @ 2018 . All rights reserved. 
miR-98, we performed a dual-luciferase reporter assay. With the aid of TargetScan ${ }^{32}$, we predicted putative binding sites located in the 3'-UTR of Fas mRNA (Figure 3A). We co-transfected either control or miR-98 mimics with luciferase vectors bearing WT or MUT Fas 3'-UTR target sequences into 293T cells and measured luciferase activity. As expected, miR-98 mimics significantly decreased luciferase activity in the WT group but had no effect on the MUT counterpart (Figure 3B), suggesting that the inhibitory effect of miR-98 was mediated by binding to the predicted sites. Taken together, our results demonstrated that miR-98 directly targets the Fas 3'-UTR and suggested the potential involvement of miR-98 in the Fas-mediated apoptotic pathway.

MiR-98 inhibitor induces Fas-mediated apoptosis in normal $C D 4+T$ cells. To further analyze the functional interaction between miR-98 and Fas in clinical samples, we assessed the effect of miR-98 inhibitor on isolated primary healthy CD4+ $\mathrm{T}$ cells. Figure 4A and 4B show that miR-98 inhibitor increased Fas expression at both mRNA and protein levels, along with increases in the expression of proteins involved in the Fas-mediated apoptotic pathway. Because the FasL/Fas

\section{A}

SV40 Promoter Luciferase Fas 3'UTR poly A

miR-98 3' UUGUUAUGUUGAAUGAUGGAGU 5'

Fas 3'UTR-WT 5'...AAAUAAGGCUCUACCUCA...3' Fas 3'UTR-MUT 5'...AAAUAAGGCUGUTCGUGA...3'

B

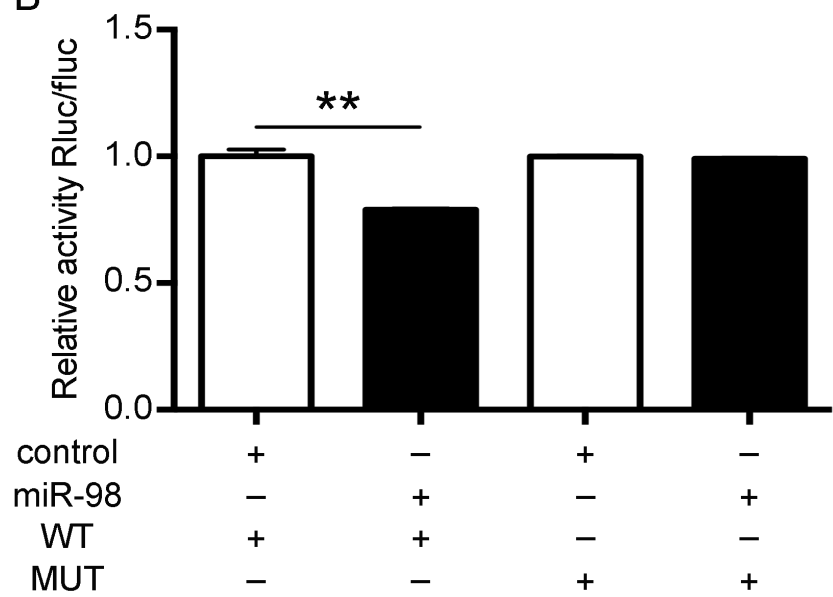

Figure 3. MiR-98 directly targets the $3^{\prime}$-UTR of Fas. A. Schematic presentation of the potential miR-98 binding sites in the $3^{\prime}$-UTR of Fas. B. Luciferase vectors were generated by inserting the wild-type (WT) or mutated (MUT) 3'-UTR fragments of Fas position 879-886 into a psiCHECK-2 plasmid. Normalized luciferase activity was assessed $48 \mathrm{~h}$ after transfection. Data shown are representative of 3 independent experiments (mean \pm SEM). **p $<0.01$. miR-98: microRNA98. pathway is the major inducer of $\mathrm{T}$ cell apoptosis, we attempted to investigate whether miR-98 could regulate Fas-mediated apoptosis by flow cytometry analysis. As shown in Figure 4D and 4E, inhibition of endogenous miR-98 increased the percentage of apoptotic cells in healthy CD4+ T cells, which resembled an "SLE-like" phenotype. In short, our results indicated that alterations in miR-98 could regulate Fas-mediated apoptosis in primary healthy CD4+ T cells.

MiR-98 mimics ameliorate Fas-mediated apoptosis in CD4+ $T$ cells from patients with SLE. Because the endogenous miR-98 level in SLE CD4+ T cells was lower than that in healthy CD4+ T cells (Figure 1A), we next examined the functional role of miR-98 mimics in SLE CD4+ T cells. As expected, the expression of Fas and Fas-mediated apoptotic proteins (cleaved/total caspase-3 and cleaved/total caspase-8) was markedly downregulated by miR-98 mimic transfection, whereas cleaved/total caspase-9, Bax, and Bcl-2 expression was almost unchanged (Figure 5A, 5B, and 5C). In addition, flow cytometry was used to validate the protective role of miR-98 in Fas-mediated apoptosis in SLE CD4+ T cells. As shown in Figure 5D, miR-98 mimic treatment decreased the apoptosis percentage. To further investigate whether the effects of miR-98 were mediated through Fas, we transfected control vector or Fas expression vector into SLE CD4+ T cells using a lentiviral transfection system, which overexpressed miR-98 and analyzed the effects of Fas overexpression (LV-Fas) on cell apoptosis. Dramatically, the results indicated that Fas reversed the function of miR-98 overexpression, which suppressed apoptosis in SLE CD4+ T cells (Figure 5E). These results indicated that miR-98 overexpression could partially ameliorate Fas-mediated cell apoptosis in patients with SLE and could be used for SLE treatment.

\section{DISCUSSION}

T cell apoptosis is well documented in SLE and regarded as a predominant factor leading to the release of autoantigens that may cause autoimmunity ${ }^{8,33}$. Elucidating the underlying molecular mechanisms and identifying effective therapeutic targets are critical for inhibiting apoptosis and relieving the associated cell dysfunction in SLE. MiR-98 was examined in our study because of its potential role in the CD4+ T cell-mediated immune response and its close association with apoptosis, according to previous studies $26,27,28$. However, little information is available regarding miR-98 in the regulation of apoptosis in SLE CD4+ T cells.

Mounting evidence suggests the involvement of aberrant expression of miRNA in the pathogenesis of $\mathrm{SLE}^{34}$. In our present study, the expression of miR-98 in freshly isolated peripheral SLE CD4+ T cells was remarkably lower than that in the CD4+ T cells of healthy donors. Decreased miR-98 expression was negatively correlated with clinical disease activity, indicating that miR-98 may serve as a new disease

Personal non-commercial use only. The Journal of Rheumatology Copyright @ 2018 . All rights reserved. 
A

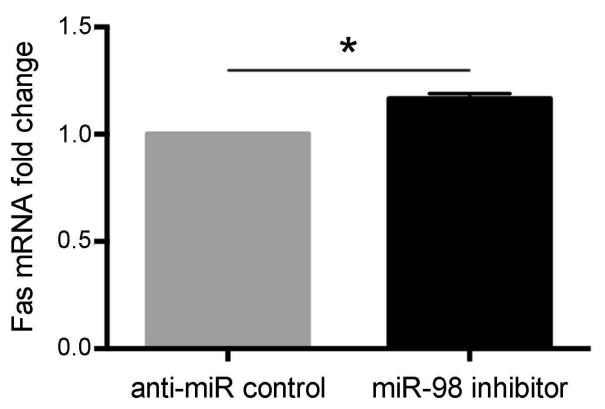

D

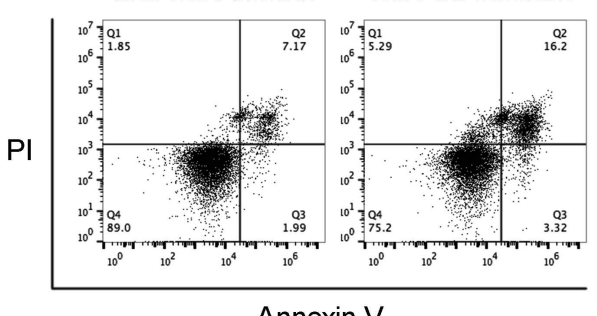

Annexin V
B

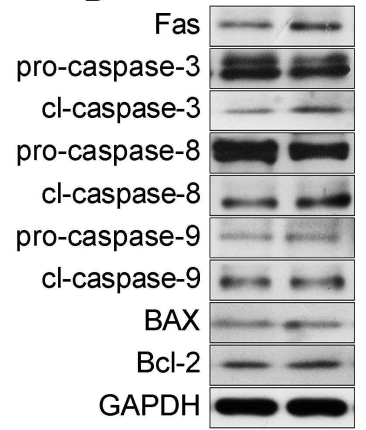

anti-miR miR-98 control inhibitor

E

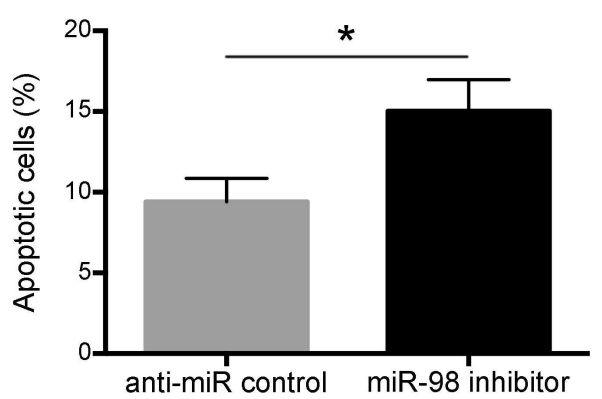

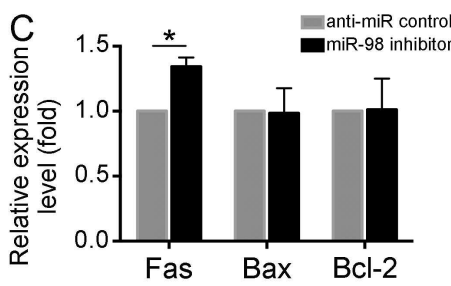

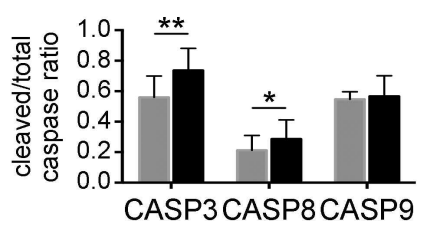

Figure 4. MiR-98 inhibitor induces Fas-mediated apoptosis in normal CD4+ T cells. A. Analysis by qRT-PCR of Fas mRNA expression in CD4+ T cells from healthy donors $(n=3) 48 \mathrm{~h}$ after transfection with miR-98 inhibitor. B. Western blot analysis of Fas and apoptosis-related protein expression in CD4+ T cells from healthy donors $(\mathrm{n}=3) 48 \mathrm{~h}$ after transfection with miR-98 inhibitor. C. Histograms show the fold changes in Fas, Bax, and Bcl-2 protein expression and the ratio of cleaved/total caspase-3, 8 , and 9 protein expression after transfection with miR-98 inhibitor. D. Apoptosis levels were measured using annexin V/PI staining and flow cytometry in CD4+ T cells from healthy donors $(n=4) 48 \mathrm{~h}$ after transfection with miR-98 inhibitor. E. Percentage of annexin V-positive apoptotic cells. Data shown are representative of 3 or more independent experiments (mean \pm SEM). ${ }^{*} \mathrm{p}<0.05$. $* * \mathrm{p}<0.01$. miR-98: microRNA98; qRT-PCR: quantitative reverse transcription PCR.

biomarker. These results are in line with previous studies, which showed that miR-98 is significantly downregulated in the CD4+ T cells from SLE patients with skin lesions and also chronic renal pathology $\mathrm{y}^{35}$, and in the PBMC from patients with active $\mathrm{SLE}^{36}$. Despite the clear influence of female sex on the risk of autoimmunity and SLE disease activity, the underlying mechanisms remain poorly understood. So far, a few miRNA have been reported to be sex-differentially expressed or responsive to sex hormones, and might contribute to the etiopathogenesis of $\mathrm{SLE}^{37}$. Among these is miR-98, which is localized to chromosome X. MiR-98 is proved to be downregulated after estrogen treatment ${ }^{38}$, and to be associated with sex differences in rheumatoid arthritis and SLE ${ }^{39,40}$. In addition, miR-98 is lower in PBMC from healthy women than in those from men $^{38}$. The activation of interferon (IFN)- $\alpha$ signaling is enhanced by $17 \beta$-estradiol by downregulating miR-98 expression, which provides an alternative perspective for understanding the mechanism underlying the sex difference in $\mathrm{SLE}^{38}$. Thus, we sought to identify whether miR-98 contributes to the disproportionate prevalence of SLE in women. However, in our study, miR-98 expression in CD4+ $\mathrm{T}$ cells was weakly correlated with $\operatorname{sex}(\mathrm{p}=0.8875$, data not shown). In addition to the different lymphocyte subsets studied, we attribute this to the small sample size (female: $n=37$, male: $n=3$ ), because women of childbearing age develop SLE about 9 times more often than men do. Moreover, in our study we also observed a high IFN signature in SLE CD4+ T cells; of note, miR-98 correlated positively with $O A S L$ mRNA expression $(\mathrm{r}=0.7347, \mathrm{p}=0.0006)$, but not with expression of the other 4 IFN-inducible genes ${ }^{41}$ (MX1, OAS1, ISG15, and LY6E; data not shown). The exact role of $O A S L$ in SLE in a larger sample size deserves to be studied in more depth. Further, miR-98 negatively regulates interleukin (IL)-10 production in macrophages after lipopolysaccharide (LPS) stimulation ${ }^{42}$, PBMC from patients with SLE produce much higher levels of IL-10 than do those from healthy controls ${ }^{43}$, and increased serum IL-10 in patients with SLE promotes apoptosis of $\mathrm{T}$ cell subsets through the caspase- 8 pathway initiated by Fas signaling 44 . These findings all suggest that miR-98 is indeed involved in the pathogenesis of SLE.

Cell apoptosis mainly occurs through 2 pathways. The extrinsic pathway is triggered by the binding of FasL to Fas, leading to caspase- 8 and caspase- 3 activation, which initiates multiple pro-apoptotic processes ${ }^{45}$. The intrinsic pathway,

Personal non-commercial use only. The Journal of Rheumatology Copyright @ 2018 . All rights reserved. 
A

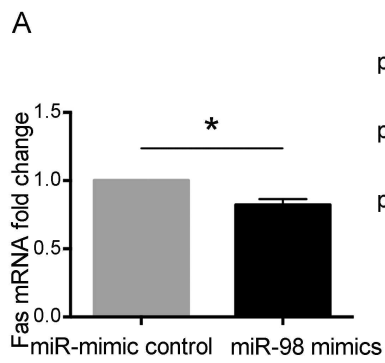

D

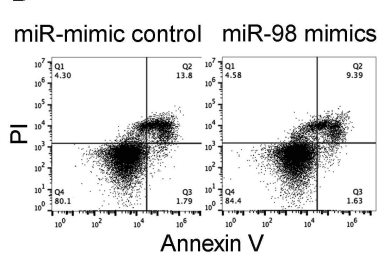

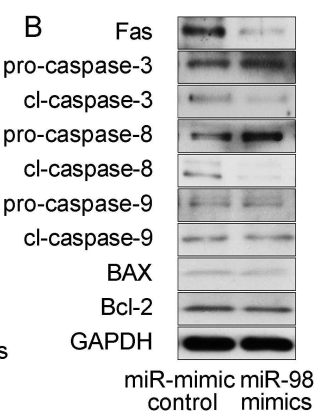

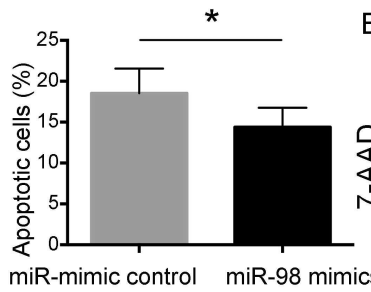

C
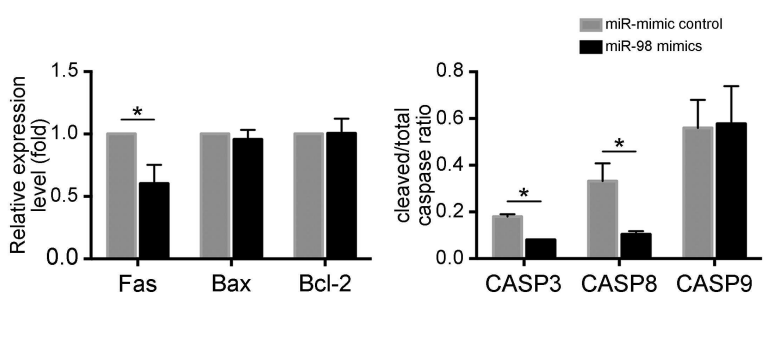

E
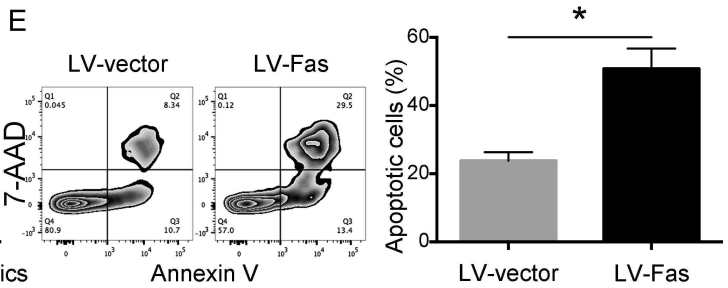

Figure 5. MiR-98 mimics ameliorate Fas-mediated apoptosis in CD4+ T cells from patients with SLE. A. Analysis by qRT-PCR of Fas mRNA expression in CD4+ T cells from patients with SLE $(n=4) 48 \mathrm{~h}$ after transfection with miR-98 mimics. B. Western blot analysis of Fas and apoptosis-related protein expression in CD4+ T cells from patients with SLE $(\mathrm{n}=4) 48 \mathrm{~h}$ after transfection with miR-98 mimics. C. Fold changes in Fas, Bax, and Bcl-2 protein expression and the ratio of cleaved/total caspase-3, 8 , and 9 protein expression after transfection with miR-98 mimics. D. Apoptosis levels were measured using FITC-Annexin V/PI staining and flow cytometry in CD4+ T cells from patients with SLE $(n=6) 48 \mathrm{~h}$ after transfection with miR-98 mimics. Graph indicates the percentage of annexin $\mathrm{V}$-positive apoptotic cells. E. SLE CD4+ T cells $(\mathrm{n}=3)$ were infected with LV-Fas or LV-vector for $72 \mathrm{~h}$ and then transfected with miR-98 mimics for $48 \mathrm{~h}$, followed by APC-Annexin V/7-AAD staining and flow cytometry. Graph indicates the percentage of annexin $\mathrm{V}$-positive apoptotic cells. Data shown are representative of 3 or more independent experiments $(\mathrm{mean} \pm \mathrm{SEM})$. ${ }^{*} \mathrm{p}<0.05$. miR-98: microRNA98; qRT-PCR: quantitative reverse transcription PCR; APC: allophycocyanin; SLE: systemic lupus erythematosus.

which involves the activation of pro-apoptotic Bcl-2 family members, is characterized by the release of cytochrome $\mathrm{C}$ from the mitochondria, resulting in the activation of downstream caspase- 9 and caspase- $3^{46}$. We simultaneously examined whether these 2 pathways were involved in miR-98-mediated $\mathrm{T}$ cell protection.

First, we analyzed the expression of death receptor member Fas in clinical samples and found that the expression of Fas was dramatically upregulated in SLE CD4+ T cells, in agreement with previous studies ${ }^{21,22}$. MiR-98 significantly reversed the expression of Fas mRNA and protein. Moreover, Western blotting showed that the ratio of cleaved/total caspase- 8 and cleaved/total caspase- 3 in Jurkat and SLE CD4+ $\mathrm{T}$ cells was markedly downregulated by miR-98 mimic transfection, whereas anti-miR-98 restored their expression in Jurkat and healthy CD4+ T cells. Further, an annexin $\mathrm{V}$ staining assay showed that upregulation of miR-98 by mimic transfection protected SLE CD4+ T cells against Fas-mediated apoptosis, while miR-98 inhibitor had the completely opposite effect. Therefore, we demonstrated that miR-98 modulates the extrinsic pathway of apoptosis in Jurkat and CD4+ T cells. This finding is consistent with previously reported experiments that showed that glucocorticoids inhibited Fas expression through the induction of miR-98 and suggested that enhancing the expression of miR-98 in SLE might have clinical benefits ${ }^{47}$. However, Bijl, et al failed to find any differences in Fas expression between CD4+ T cells from patients with SLE and those from healthy controls $^{48}$. In addition to the strong heterogeneity in the etiology and symptoms of SLE pathology, this discrepancy might be due to methodological differences (Bijl, et al performed multicolor flow cytometry to evaluate membrane expression of Fas on CD4+ T cells, whereas we isolated CD4+ $\mathrm{T}$ cells by magnetic separation and performed qRT-PCR and Western blotting to detect total mRNA and protein expression of Fas) or the different patient cohorts studied (different race, sex, age, environmental factors, disease activity, the use of medication, and so on).

Another apoptosis signaling pathway is mediated by the Bcl-2 protein family, which includes pro-apoptotic members (Bax, Bak, and Bim) and anti-apoptotic members (Bcl-2, $\mathrm{Bcl}-\mathrm{xl}$, and Mcl-1). Our results showed that the expression of Bcl-2 and Bax was neither affected by miR-98 mimic transfection nor by miR-98 inhibitor. Therefore, we speculated that miR-98 has limited effects on the intrinsic pathway of CD4+ T cell apoptosis in SLE.

Even as miR-98 has emerged as one of the regulators of apoptosis in CD4+ T cells, the mechanism of the decreased expression of miR-98 in SLE is not clear. It was reported that miR-98 was downregulated after estrogen or high glucose treatment ${ }^{38,49}$ or following LPS stimulation ${ }^{42}$, but was upregulated in a hypoxia-inducible factor- $1 \alpha$-dependent manner ${ }^{50}$

Personal non-commercial use only. The Journal of Rheumatology Copyright (C) 2018. All rights reserved. 
or by glucocorticoid ${ }^{47}$. In addition to the above potential environmental (exogenous) factors, it would also be of interest to investigate the genetic (endogenous) factors, such as methylation status of the miR-98 gene in SLE and transcription factors that bind to the promoter region of miR-98, which could clarify the mechanism of the defective expression of miR-98 and the subsequent loss of immune tolerance in patients with SLE.

Our findings revealed that miR-98 is important in the pathogenesis of SLE. MiR-98 downregulation contributes to the dysregulation of apoptosis in SLE, partially through direct interaction with the Fas 3'-UTR, resulting in aberrant T cell responses. Increased expression of miR-98 may benefit the maintenance of T-lymphocyte homeostasis under physiological conditions, and miR-98 oligonucleotides might be helpful for relieving Fas-mediated apoptosis in SLE. Thus, modulation of miR-98 is a novel therapeutic strategy for this disease. Further research investigating the possible causes of the downregulation of miR-98 in SLE is required. Moreover, additional investigation on transgenic animal models (such as treating SLE mice with miR-98 agomir) would help us to identify the in vivo function of miR-98.

\section{REFERENCES}

1. Crispin JC, Liossis SN, Kis-Toth K, Lieberman LA, Kyttaris VC, Juang YT, et al. Pathogenesis of human systemic lupus erythematosus: recent advances. Trends Mol Med 2010;16:47-57.

2. Horwitz DA. Peripheral blood CD4+ T cells in systemic lupus erythematosus. Lupus 2001;10:319-20.

3. Bouzahzah F, Jung S, Craft J. CD4+ T cells from lupus-prone mice avoid antigen-specific tolerance induction in vivo. J Immunol 2003;170:741-8.

4. Chang NH, MacLeod R, Wither JE. Autoreactive B cells in lupus-prone New Zealand black mice exhibit aberrant survival and proliferation in the presence of self-antigen in vivo. J Immunol 2004; 172:1553-60.

5. Moulton VR, Tsokos GC. T cell signaling abnormalities contribute to aberrant immune cell function and autoimmunity. J Clin Invest 2015;125:2220-7.

6. Gupta S, Kaplan MJ. The role of neutrophils and NETosis in autoimmune and renal diseases. Nat Rev Nephrol 2016;12:402-13.

7. Lood C, Blanco LP, Purmalek MM, Carmona-Rivera C, De Ravin SS, Smith CK, et al. Neutrophil extracellular traps enriched in oxidized mitochondrial DNA are interferogenic and contribute to lupus-like disease. Nat Med 2016;22:146-53.

8. Munoz LE, van Bavel C, Franz S, Berden J, Herrmann M, van der Vlag J. Apoptosis in the pathogenesis of systemic lupus erythematosus. Lupus 2008;17:371-5.

9. Wu H, Fu S, Zhao M, Lu L, Lu Q. Dysregulation of cell death and its epigenetic mechanisms in systemic lupus erythematosus. Molecules 2016 Dec 27;22.

10. Emlen W, Niebur J, Kadera R. Accelerated in vitro apoptosis of lymphocytes from patients with systemic lupus erythematosus. J Immunol 1994;152:3685-92.

11. Shah D, Aggarwal A, Bhatnagar A, Kiran R, Wanchu A. Association between $\mathrm{T}$ lymphocyte sub-sets apoptosis and peripheral blood mononuclear cells oxidative stress in systemic lupus erythematosus. Free Radic Res 2011;45:559-67.

12. Dhir V, Singh AP, Aggarwal A, Naik S, Misra R. Increased T-lymphocyte apoptosis in lupus correlates with disease activity and may be responsible for reduced T-cell frequency: a cross-sectional and longitudinal study. Lupus 2009;18:785-91.

13. Lorenz HM, Grunke M, Hieronymus T, Herrmann M, Kuhnel A, Manger B, et al. In vitro apoptosis and expression of apoptosis-related molecules in lymphocytes from patients with systemic lupus erythematosus and other autoimmune diseases. Arthritis Rheum 1997;40:306-17.

14. Silvestris F, Frassanito MA, Cafforio P, Potenza D, Di Loreto M, Tucci M, et al. Antiphosphatidylserine antibodies in human immunodeficiency virus-1 patients with evidence of T-cell apoptosis and mediate antibody-dependent cellular cytotoxicity. Blood 1996;87:5185-95.

15. Alarcon-Segovia D, Llorente L, Ruiz-Arguelles A. The penetration of autoantibodies into cells may induce tolerance to self by apoptosis of autoreactive lymphocytes and cause autoimmune disease by dysregulation and/or cell damage. J Autoimmun 1996;9:295-300.

16. Trauth BC, Klas C, Peters AM, Matzku S, Moller P, Falk W, et al. Monoclonal antibody-mediated tumor regression by induction of apoptosis. Science 1989;245:301-5.

17. Nagata S, Golstein P. The Fas death factor. Science 1995; 267:1449-56

18. Suda T, Takahashi T, Golstein P, Nagata S. Molecular cloning and expression of the Fas ligand, a novel member of the tumor necrosis factor family. Cell 1993;75:1169-78.

19. Ju ST, Panka DJ, Cui H, Ettinger R, el-Khatib M, Sherr DH, et al. Fas(CD95)/FasL interactions required for programmed cell death after T-cell activation. Nature 1995;373:444-8.

20. Ohsako S, Hara M, Harigai M, Fukasawa C, Kashiwazaki S. Expression and function of Fas antigen and bcl-2 in human systemic lupus erythematosus lymphocytes. Clin Immunol Immunopathol 1994;73:109-14.

21. Amasaki Y, Kobayashi S, Takeda T, Ogura N, Jodo S, Nakabayashi T, et al. Up-regulated expression of Fas antigen (CD95) by peripheral naive and memory $\mathrm{T}$ cell subsets in patients with systemic lupus erythematosus (SLE): a possible mechanism for lymphopenia. Clin Exp Immunol 1995;99:245-50.

22. Xue C, Lan-Lan W, Bei C, Jie C, Wei-Hua F. Abnormal Fas/FasL and caspase-3-mediated apoptotic signaling pathways of $\mathrm{T}$ lymphocyte subset in patients with systemic lupus erythematosus. Cell Immunol 2006;239:121-8.

23. Knipping E, Krammer PH, Onel KB, Lehman TJ, Mysler E, Elkon KB. Levels of soluble Fas/APO-1/CD95 in systemic lupus erythematosus and juvenile rheumatoid arthritis. Arthritis Rheum 1995;38:1735-7.

24. van Lopik T, Bijl M, Hart M, Boeije L, Gesner T, Creasy AA, et al. Patients with systemic lupus erythematosus with high plasma levels of sFas risk relapse. J Rheumatol 1999;26:60-7.

25. Liphaus BL, Kiss MH, Carrasco S, Goldenstein-Schainberg C. Increased Fas and Bcl-2 expression on peripheral mononuclear cells from patients with active juvenile-onset systemic lupus erythematosus. J Rheumatol 2007;34:1580-4.

26. Johnson CD, Esquela-Kerscher A, Stefani G, Byrom M, Kelnar K, Ovcharenko D, et al. The let-7 microRNA represses cell proliferation pathways in human cells. Cancer Res 2007; 67:7713-22.

27. Wang S, Tang Y, Cui H, Zhao X, Luo X, Pan W, et al. Let-7/miR-98 regulate Fas and Fas-mediated apoptosis. Genes Immun 2011;12:149-54.

28. Sun C, Liu H, Guo J, Yu Y, Yang D, He F, et al. MicroRNA-98 negatively regulates myocardial infarction-induced apoptosis by down-regulating Fas and caspase-3. Sci Rep 2017;7:7460.

29. Qin H, Zhu X, Liang J, Wu J, Yang Y, Wang S, et al. MicroRNA-29b contributes to DNA hypomethylation of CD4+ T cells in systemic lupus erythematosus by indirectly targeting DNA methyltransferase 1. J Dermatol Sci 2013;69:61-7. 
30. Xie L, Yang R, Liu S, Lyle S, Cotsarelis G, Xiang L, et al. TR3 is preferentially expressed by bulge epithelial stem cells in human hair follicles. Lab Invest 2016;96:81-8.

31. Wang H, Xu J, Ji X, Yang X, Sun K, Liu X, et al. The abnormal apoptosis of $\mathrm{T}$ cell subsets and possible involvement of IL-10 in systemic lupus erythematosus. Cell Immunol 2005;235:117-21.

32. Agarwal V, Bell GW, Nam JW, Bartel DP. Predicting effective microRNA target sites in mammalian mRNAs. Elife 2015 Aug 12;4.

33. Huggins ML, Todd I, Cavers MA, Pavuluri SR, Tighe PJ, Powell RJ. Antibodies from systemic lupus erythematosus (SLE) sera define differential release of autoantigens from cell lines undergoing apoptosis. Clin Exp Immunol 1999;118:322-8.

34. Shen N, Liang D, Tang Y, de Vries N, Tak PP. MicroRNAs-novel regulators of systemic lupus erythematosus pathogenesis. Nat Rev Rheumatol 2012;8:701-9.

35. Zhao M, Liu S, Luo S, Wu H, Tang M, Cheng W, et al. DNA methylation and mRNA and microRNA expression of SLE CD4+ T cells correlate with disease phenotype. J Autoimmun 2014; 54:127-36.

36. Stagakis E, Bertsias G, Verginis P, Nakou M, Hatziapostolou M, Kritikos $\mathrm{H}$, et al. Identification of novel microRNA signatures linked to human lupus disease activity and pathogenesis: miR-21 regulates aberrant $\mathrm{T}$ cell responses through regulation of PDCD4 expression. Ann Rheum Dis 2011;70:1496-506.

37. Khan D, Dai R, Ansar Ahmed S. Sex differences and estrogen regulation of miRNAs in lupus, a prototypical autoimmune disease. Cell Immunol 2015;294:70-9

38. Dong G, Fan H, Yang Y, Zhao G, You M, Wang T, et al. 17beta-Estradiol enhances the activation of IFN-alpha signaling in B cells by down-regulating the expression of let-7e-5p, miR-98-5p and miR-145a-5p that target IKKepsilon. Biochim Biophys Acta 2015;1852:1585-98.

39. Khalifa O, Pers YM, Ferreira R, Senechal A, Jorgensen C, Apparailly F, et al. X-linked miRNAs associated with gender differences in rheumatoid arthritis. Int J Mol Sci 2016 Nov 8;17.
40. Hewagama A, Gorelik G, Patel D, Liyanarachchi P, McCune WJ, Somers E, et al. Overexpression of X-linked genes in T cells from women with lupus. J Autoimmun 2013;41:60-71.

41. Feng X, Wu H, Grossman JM, Hanvivadhanakul P, FitzGerald JD, Park GS, et al. Association of increased interferon-inducible gene expression with disease activity and lupus nephritis in patients with systemic lupus erythematosus. Arthritis Rheum 2006;54:2951-62.

42. Liu Y, Chen Q, Song Y, Lai L, Wang J, Yu H, et al. MicroRNA-98 negatively regulates IL-10 production and endotoxin tolerance in macrophages after LPS stimulation. FEBS Lett 2011;585:1963-8.

43. Beebe AM, Cua DJ, de Waal Malefyt R. The role of interleukin-10 in autoimmune disease: systemic lupus erythematosus (SLE) and multiple sclerosis (MS). Cytokine Growth Factor Rev 2002; 13:403-12.

44. Yang X, Sun B, Wang H, Yin C, Wang X, Ji X. Increased serum IL-10 in lupus patients promotes apoptosis of $\mathrm{T}$ cell subsets via the caspase 8 pathway initiated by Fas signaling. J Biomed Res 2015;29:232-40.

45. Wajant $\mathrm{H}$. The Fas signaling pathway: more than a paradigm. Science 2002;296:1635-6.

46. Adams JM, Cory S. The Bcl-2 apoptotic switch in cancer development and therapy. Oncogene 2007;26:1324-37.

47. Davis TE, Kis-Toth K, Szanto A, Tsokos GC. Glucocorticoids suppress T cell function by up-regulating microRNA-98. Arthritis Rheum 2013;65:1882-90.

48. Bijl M, Horst G, Limburg PC, Kallenberg CG. Fas expression on peripheral blood lymphocytes in systemic lupus erythematosus (SLE): relation to lymphocyte activation and disease activity. Lupus 2001;10:866-72.

49. Li XX, Liu YM, Li YJ, Xie N, Yan YF, Chi YL, et al. High glucose concentration induces endothelial cell proliferation by regulating cyclin-D2-related miR-98. J Cell Mol Med 2016;20:1159-69.

50. Li HW, Meng Y, Xie Q, Yi WJ, Lai XL, Bian Q, et al. miR-98 protects endothelial cells against hypoxia/reoxygenation induced-apoptosis by targeting caspase-3. Biochem Biophys Res Commun 2015;467:595-601. 